\title{
Local delivery of T-bet shRNA reduces inflammation in collagen II-induced arthritis via downregulation of IFN- $\gamma$ and IL-17
}

\author{
YUAN XUE ${ }^{1,2}$, YONG YANG ${ }^{3}$, ZHAOLIANG SU ${ }^{1}$, PRINCE AMOATT BARNIE ${ }^{1}$, \\ DONG ZHENG $^{1}$, YUN ZHANG ${ }^{1}$, YAN XU ${ }^{1}$, SHENGJUN WANG ${ }^{1}$ and HUAXI XU ${ }^{1}$ \\ ${ }^{1}$ Department of Immunology, Institute of Laboratory Medicine, Jiangsu University, Zhenjiang, \\ Jiangsu 212013; ${ }^{2}$ Zhenjiang Center for Disease Prevention and Control, Zhenjiang, Jiangsu 212000; \\ ${ }^{3}$ The Affiliated People's Hospital of Jiangsu University, Zhenjiang, Jiangsu 212001, P.R. China
}

Received June 19, 2013; Accepted January 6, 2014

DOI: $10.3892 / \mathrm{mmr} .2014 .1893$

\begin{abstract}
Th1 and Th17 cells are involved in the pathogenesis of rheumatoid arthritis (RA). T-bet, a Th1-specific transcription factor, appears to drive the maturation of Th1 and IFN- $\gamma$ secretion. In the present study, we established the T-bet shRNA recombinant plasmid (p-T-shRNA) and explored its possible anti-inflammatory effect in a collagen-induced arthritis (CIA) model by local injection of plasmid vectors. For the initiation of CIA, DBA/1J mice were immunized with type II collagen (CII) in Freund's adjuvant and the CII-immunized mice were treated with p-T-shRNA. Levels of T-bet, IFN- $\gamma$, IL-17 and ROR $\gamma \mathrm{t}$ mRNA in splenocytes and synovial joints were measured by quantitative real-time PCR and T-bet expression in joint tissue was detected by immunohistochemistry staining. The intracellular IFN- $\gamma$ and IL-17 were analyzed by flow cytometry (FCM). The results demonstrated that therapeutic administration on the local joints with p-T-shRNA significantly suppressed IFN- $\gamma$ and IL-17 gene expression and improved the pathogenesis of arthritis in CIA mice, while administration of a plasmid expressing T-bet (pIRES-T-bet) accelerated the disease onset. Our study suggests that $T$-bet may be developed as a potential target for arthritis therapy.
\end{abstract}

\section{Introduction}

Rheumatoid arthritis (RA) is a chronic inflammatory disease that mainly affects the human synovial membrane, cartilage and bone. Approximately $1 \%$ of the population suffer from RA

Correspondence to: Professor Huaxi Xu or Professor Shengjun Wang, Department of Immunology, Institute of Laboratory Medicine, Jiangsu University, 301 Xuefu Road, Zhenjiang, Jiangsu 212013, P.R. China E-mail:xuhx@ujs.edu.cn

E-mail: sjwjsu@yahoo.com.cn

Key words: T-bet, shRNA, IFN- $\gamma$, IL-17, collagen-induced arthritis and it is associated with significant morbidity and increased mortality. Several studies have demonstrated that T lymphocytes, B lymphocytes, mast cells and other immune cells are critical in the pathogenesis and progression of inflammation. Several studies on spontaneous animal models of arthritis and cell-cell interactions have implicated $\mathrm{T}$ cells in driving synovial inflammation and joint destruction (1-5), and Th1 cells are elevated as a dominant cell in RA. T-bet is a specific Th1 transcriptional factor, which contributes to the differentiation of Th1 and is required for the generation of IFN- $\gamma$-producing Th1 cells (6-7). Th17 cells are also important for the modulation of autoimmune responses. IL-17 secreted by Th17 is critical in the pathogenesis of RA and it is capable of promoting inflammation by inducing a variety of proinflammatory mediators and inducing bone and cartilage destruction (8-11). Recent studies have demonstrated that T-bet has an effect on Th1 and Th17 cells (12) and is important in experimental autoimmune encephalomyelitis and RA (13-14). Collagen induced arthritis (CIA) is the most useful animal model for studying RA in humans (15). In the present study, we created a chicken type II CIA animal model, established the T-bet shRNA recombinant plasmid (p-T-shRNA) and examined its possible anti-inflammatory effect in the CIA model by local injection of plasmid vectors.

\section{Materials and methods}

Animals and CIA model induction. Male, 8 to 10-week-old DBA/1LacJ mice (Shanghai SLAC Laboratory Animal Co., Ltd., Shanghai, China) were used and $100 \mu \mathrm{g}$ of chicken type II collagen (CII; Sigma-Aldrich, St. Louis, MO, USA) was injected intradermally at the base of the tail, which was emulsified in equal volumes of Freund's complete adjuvant (2 $\mathrm{mg} / \mathrm{ml}$; Mycobacterium tuberculosis). The study was approved by the Ethics Committee of Jiangsu University.

CII-immunized mice were divided into five groups and then injected in the joint every $48 \mathrm{~h}$ with PBS buffer, pIRES-T-bet, pIRES empty vector, p-T-shRNA or p-nonsense-shRNA, respectively, from day 1 to 20. Starting at the first day following the first CII immunization, the mice 
were examined for the development and severity of arthritis until the 50th day. Disease severity was scored on a scale from 0 to 4 by visual inspection of paws and the score criteria were as follows: 0, no evidence of erythema and swelling; 1 , erythema and mild swelling confined to the mid-foot (tarsals) or ankle joint; 2, erythema and mild swelling extended from the ankle to the mid-foot; 3 , erythema and moderate swelling extended from the ankle to the metatarsal joints; 4, erythema and severe swelling encompassed the ankle, foot and digits.

$H \& E$ and immunohistochemistry staining. Mouse knee joints were fixed in $10 \%$ buffered formalin in PBS overnight, decalcified in $10 \%$ formic acid in water for $24 \mathrm{~h}$, embedded in paraffin and sectioned at $5 \mu \mathrm{m}$ thickness prior to staining with H\&E. Sections for immunochemistry staining were immersed in $0.01 \mathrm{M}$, pH 6.0 citrate buffer (30 min) in a water bath maintained at $100^{\circ} \mathrm{C}$ for antigen retrieval. Hydrogen peroxide (3\%) in methanol (30 min at room temperature) was used to deactivate endogenous peroxidase. Sections were washed in PBS 3 times and $30 \mathrm{mg} / \mathrm{ml}$ BSA was added (30 min at room temperature) for the closure of non-specific binding sites. Thereafter, the sections were exposed to rabbit anti-T-bet antibody (Santa Cruz Biotechnology, Inc., Santa Cruz, CA, USA), 1:200 diluted in $30 \mathrm{mg} / \mathrm{ml}$ BSA, at $4^{\circ} \mathrm{C}$ overnight in a wet box followed by staining with a MaxVision ${ }^{\mathrm{TM}}$ kit (kit-5020; MaiXin Technology Co., Ltd., Shenzhen, Guangdong, China), then stained with freshly prepared diaminobenzidine solution and counterstained with hematoxylin. Sections were dehydrated with a graded series of alcohol, vitrified by dimethylbenzene and covered with neutral gum. Results were observed and analyzed using a Leica fluorescence microscope and image analysis system (Leica, Mannheim, Germany). Section assessments were conducted by two blinded investigators for mean inflammation, pannus formation, cartilage damage, bone damage and T-bet expression.

Quantitative real-time PCR. Total RNA was isolated from mice splenocytes or joint tissue with TRIzol reagent (Invitrogen Life Technologies, Carlsbad, CA, USA) and the joint was homogenated. T-bet, IFN- $\gamma$, ROR $\gamma$ t and IL-17 mRNA in splenocytes and synovial joints were measured by quantitative real-time PCR.

Quantitative real-time PCR was performed with the SYBR green 1 two-step qRT-PCR kit with ROX (Invitrogen Life Technologies) according to the manufacturer's instructions. The threshold cycle (CT) of gene products was determined and set to the log linear range of the amplification curve and kept constant. Relative expression was calculated with normalization to $\beta$-actin values. The sequences of the primers were designed to span at least one intron. The primers used were as follows: T-bet sense, 5'-ATGCCAGGGAACCGCTTAT-3' and antisense, 5'-CAGATGCGTACATGGACTCAAA-3'; IFN- $\gamma$ sense, 5'-AAGCGTCATTGAATCACACC-3' and antisense, 5'-CGAAATCAGCAGCGACTCCTTAG-3'; IL-17 sense, 5'-GGTCAACCTCCAAGTCTTTAACTC-3' and antisense, 5'-TTAAAAATGCAAGTAAGTTTGCTG-3'; ROR $\gamma$ t sense, 5'-GCTCCCGGCCTGGTCTGCTC-3' and antisense, 5'-AGGTGGCGGGGTGGTTTCTGA-3'; FoxP3 sense, 5'-CCACCATATTCCAATACCTT-3' and antisense, 5'-GCTGCTTGGACCCTGTTCT-3'.

Intracellular cytokine staining. $\mathrm{CD}^{+}$cells were isolated from peripheral blood lymphocytes by magnetic-activated cell sorting (MACS) and the purity of $\mathrm{CD}^{+}$cells was routinely $95 \%$. To assess the intracellular cytokine production, $\mathrm{CD}^{+}$ lymphocytes were incubated with ionomycin $(500 \mathrm{ng} / \mathrm{ml})$ and phorbol myristate acetate (PMA; $50 \mathrm{ng} / \mathrm{ml}$; Sigma-Aldrich) in the presence of monensin $(1 \mu \mathrm{g} / \mathrm{ml}$; eBioscience, San Diego, CA, USA). The cells were washed and resuspended in $1 \%$ BSA in PBS. Following washing twice, cells were fixed and permeabilized using Cytofix/Cytoperm solution (BD Biosciences, Franklin Lakes, NJ, USA) for $20 \mathrm{~min}$ at $4^{\circ} \mathrm{C}$, and stained for FITC-IL-17 (BioLegend, San Diego, CA, USA) and PE-IFN- $\gamma$ (eBioscience) with specific Abs or isotype control according to the manufacturer's instructions. Data were acquired by flow cytometry (FCM; BD Biosciences) and analyzed using Cell Quest software (BD Biosciences).

Statistical analysis. Data were summarized as the mean \pm SD. Means were compared with the ANOVA where appropriate for statistical analysis. $\mathrm{P}<0.05$ was considered to indicate a statistically significant difference.

\section{Results}

Locally enhanced expression of the T-bet gene may promote the onset of arthritis at the joint. To determine whether T-bet acts as a driving factor for CIA development, we injected recombinant plasmid pIRES-T-bet into the hind ankle of CII-immunized mice every $48 \mathrm{~h}$ for ten-doses, starting at day 1 following the first immunization, and found an accelerated onset of arthritis (Fig. 1). The hind paw became red and swollen more quickly following pIRES-T-bet injection.

Silencing the T-bet gene may induce delayed onset of arthritis. The mice were administered with a single i.p. injection of p-T-shRNA at the time of immunization to silence the T-bet gene for long-lasting suppressive capacity on CIA induction in vivo. The results demonstrated that the expression of T-bet was effectively reduced in the joint tissue of CIA mice treated with p-T-shRNA (Fig. 1A), the incidence rate of CIA was significantly reduced and the onset of arthritis was markedly delayed (Fig. 1B).

$H \& E$ and immunohistochemistry staining. We compared the inflammatory score of three groups. The infiltrated inflammatory cells and the level of T-bet expression were different in different groups. The infiltrated inflammatory cells in the pIRES-T-bet group were similar to the CIA blank group, but higher than that in the p-T-shRNA group. The immunohistochemistry staining result demonstrated that the $T$-bet expression was higher in the pIRES-T-bet group than that in the CIA blank group, however it was decreased in the p-T-shRNA group compared with other groups. The T-bet immunopositive products were subcellularly localized in the nuclei (Fig. 2).

Expression of related inflammatory factors in the spleen or local joint. We analyzed the levels of T-bet mRNA in the 


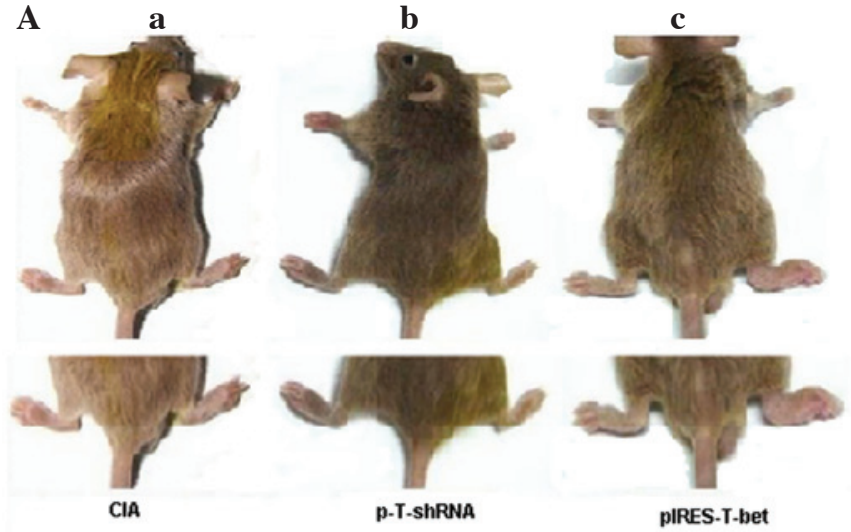

B

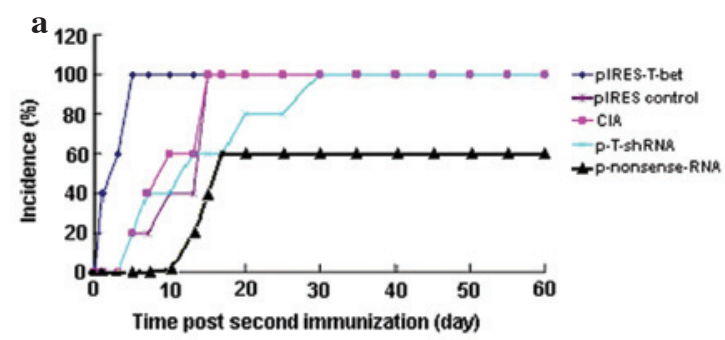

b

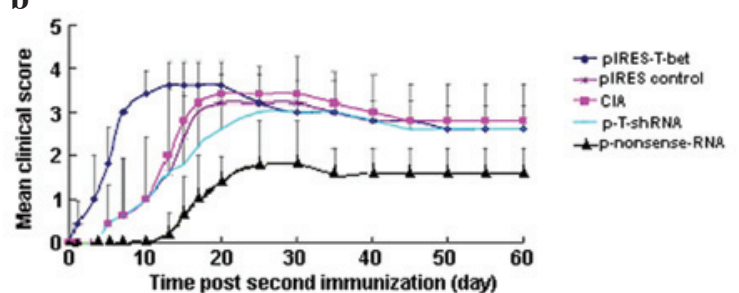

Figure 1. Effect of p-T-shRNA on the formation of CIA. (A) Injection of p-T-shRNA was able to ameliorate inflammation at the local joint, while pIRES-T-bet led to the deterioration of the disease at the joint. (Aa) CIA blank control, without treatment using p-T-shRNA or pIRES-T-bet; $(\mathrm{Ab})$ the right hind paw was administrated with p-T-shRNA and demonstrated that the treated paw was thinner than the CIA blank control; (Ac) the right hind paw, which was treated with pIRES-T-bet, was worse for swelling. (B) The incidence and clinical score of arthritis in the experimental mice following the second immunization with CII. (Ba) The CIA incidence of mice from different groups: the arthritis percentage in the mice injected with p-T-shRNA was $60 \%$ reduced, however, in the pIRES-T-bet injected mice, the incidence of CIA was $100 \%$ (similar to the CIA blank control). The time of CIA onset was $\sim 13$ days in the p-T-shRNA group and 1 day in the pIRES-T-bet group. $(\mathrm{Bb})$ The inflammatory score. There was a distinct improvement in the mice injected with p-T-shRNA compared with pIRES-T-bet or CIA blank control, however there were no apparent differences between pIRES-T-bet and the CIA blank control. CIA, collagen-induced arthritis; p-T-shRNA, T-bet shRNA recombinant plasmid; pIRES-T-bet, plasmid expressing T-bet; CII, type II collagen.

spleen and local joints from different groups and the results demonstrated that the T-bet expression was increased in the limited joints following injection of plasmid pIRES-T-bet, while it was downregulated by shRNA injection. There was no clear difference in $T$-bet expression in the spleen among the CIA blank control, pIRES-T-bet and p-T-shRNA groups. Our data indicated that the changes in IFN- $\gamma$ and IL-17 expression were consistent with the level of T-bet mRNA, implying that $T$-bet was able to affect the level of cytokines at a certain phase. The topical application of pIRES-T-bet or p-T-shRNA on joint tissue was not able to induce the reduction or increase of $T$-bet expression in the splenocytes,

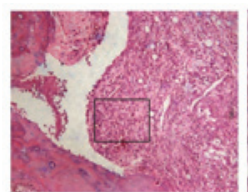

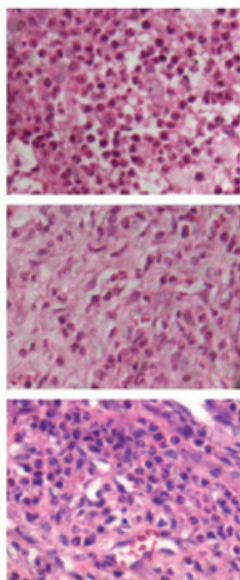

HE $(400 \times)$

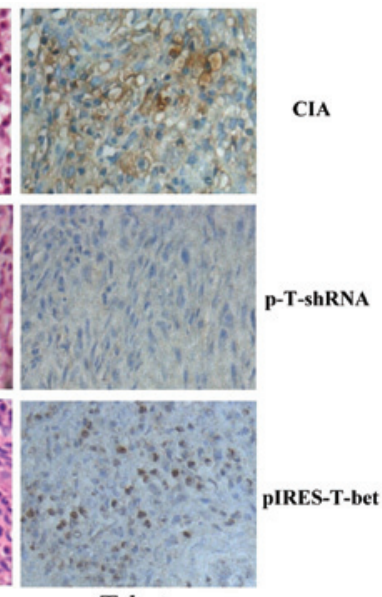

T-bet
Figure 2. Expression of T-bet and inflammatory pathology in different groups. The infiltrated cells and level of T-bet expression were significantly increased in the CIA blank control and the pIRES-T-bet group, however were significantly suppressed in the p-T-shRNA group. Although, there was no significant difference in the T-bet expression level between the CIA blank control and the pIRES-T-bet group, its subcellular distribution was completely different. In the pIRES-T-bet group, T-bet immunopositive product was mainly localized in the nuclei of inflammatory cells, while in the CIA blank control, it was dispersedly distributed in the cytoplasm. pIRES-T-bet, plasmid expressing T-bet; HE, hematoxylin and eosin.

however in the local joint, increased T-bet expression was able to increase the level of IFN- $\gamma$ and IL-17, and reduced $T$-bet expression resulted in decreased IFN- $\gamma$ and IL-17 (Fig. 3).

Expression of intracellular IFN- $\gamma$ or IL-17 from popliteal lymph nodes. The popliteal lymph nodes were collected from different groups, the $\mathrm{CD} 4^{+} \mathrm{T}$ cells were sorted and the cells were exposed to PMA and ionomycin for $4 \mathrm{~h}$, then stained with IFN- $\gamma$-PE and IL-17-FITC for FCM analysis. The result was consistent with that detected by qRT-PCR. FCM analysis for popliteal lymph nodes, which correlated with the plasmid injection demonstrated, that IL-17 and IFN- $\gamma$ were upregulated in the mice injected with pIRES-T-bet and downregulated in the p-T-shRNA group (Fig. 4).

\section{Discussion}

Recently, the study of RA has substantially advanced. Genetic and environmental factors may lead to joint destruction. RA is thought to be a disease of the immune system in which self-tolerance breaks down $(2,16)$ and numerous studies have attempted to apply immunosuppression for the treatment of RA. Immune cells, including T lymphocytes, B lymphocytes and NK cells are all involved in the process of the disease and dendritic cells are also important in the process (17-19), as well as a number of cytokines, chemokines, adhesion molecules and matrix metalloproteinases. These molecules are controlled by a limited number of transcriptional factors, including $T$-bet and $N F-\kappa B$. Previous studies have demonstrated that Th1 cells are the dominant cells in the inflammatory reaction, including RA. T-bet is essential to the development of $\mathrm{T}$ lymphocytes, directing the differentiation of Th 0 cells into Th1 or Th 2 cells $(1,6,20)$. Numerous studies 
$\mathbf{A}$

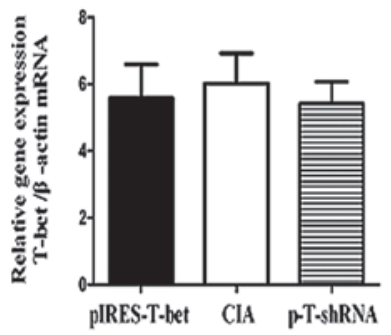

C

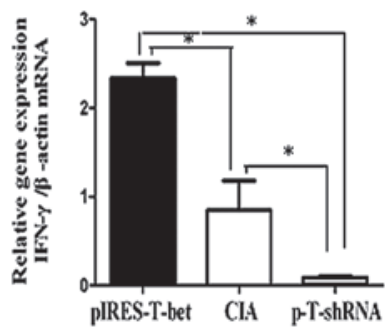

$\mathbf{E}$

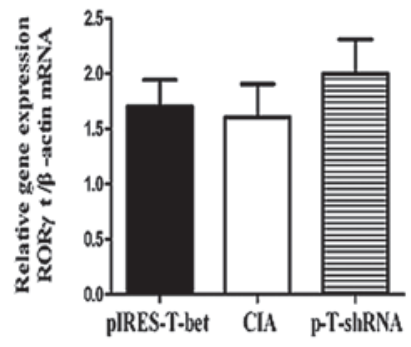

B

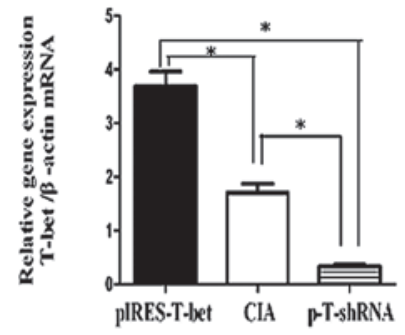

D

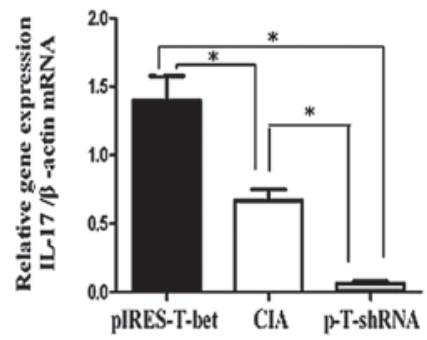

$\mathbf{F}$

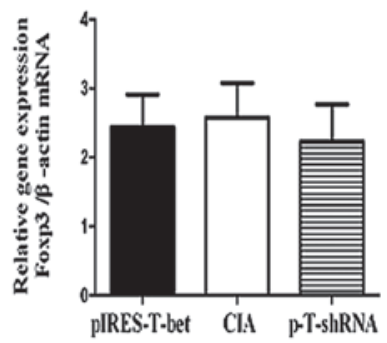

Figure 3. Effect of pIRES-T-bet or p-T-shRNA local application on the expression of inflammatory cytokines. (A) Levels of T-bet expression in splenocytes from three groups. There was no significant difference between the p-T-shRNA treated mice and pIRES-T-bet treated mice or the CIA blank control. (B) T-bet expression levels demonstrated distinct changes following local administration of pIRES-T-bet or p-T-shRNA. T-bet levels were elevated in the pIRES-T-bet group and reduced in the p-T-shRNA group. (C and D) IFN- $\gamma$ and IL-17 mRNA levels from local joints in those three groups and the two cytokines were increased in the pIRES-T-bet group, however they were reduced in the p-T-shRNA group. (E and F) The mRNA of the transcriptional factors ROR $\gamma t$ and Foxp3 were determined in the three groups in local joints, however there were no significant differences among them. "P<0.01. p-T-shRNA, T-bet shRNA recombinant plasmid; pIRES-T-bet, plasmid expressing T-bet; IFN- $\gamma$, interferon- $\gamma$; IL-17, interleukin-17; CIA, collagen-induced arthritis; ROR $\gamma$ t, RAR-related orphan receptor $\gamma$; Foxp3; forkhead box P3.

A

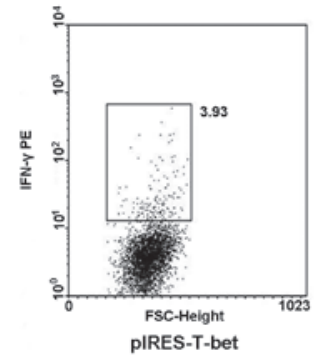

D

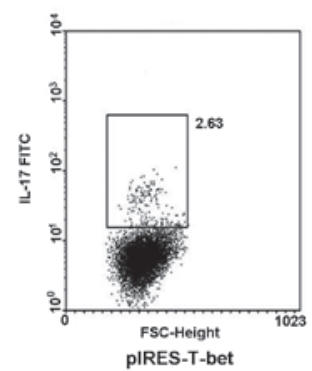

B

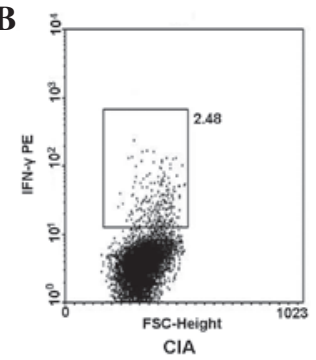

$\mathbf{E}$

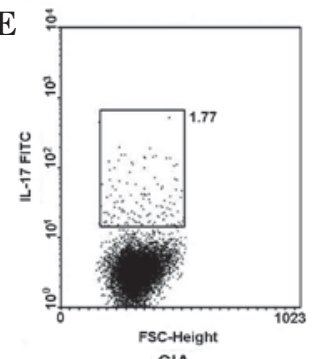

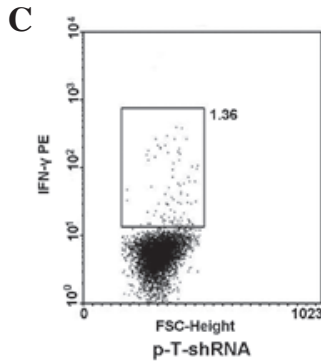

$\mathbf{F}$

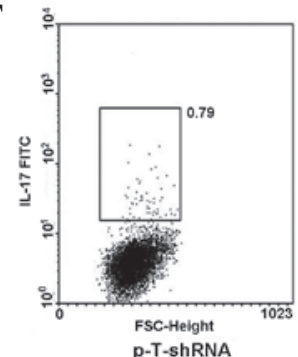

Figure 4. pIRES-T-bet or p-T-shRNA local administration affected the expression of IFN- $\gamma$ and IL-17 in PLN cells. CD4 ${ }^{+}$cells were separated from PLN by MACS and then the cultures were stimulated with PMA or ionomycin in the presence of menosin for $4 \mathrm{~h}$ and stained with fluorescent antibodies for IL-4 and IFN- $\gamma$ analysis. (A-C) IFN- $\gamma$ secreting cells (IFN- $\gamma^{+}$cells) and (D-F) exhibit the levels of IL-17 secreting cells (IL-17 cells) from three different groups, respectively and the results correlate with that of the qRT-PCR. The IFN- $\gamma^{+}$or IL-17+ cells were higher in the pIRES-T-bet group than that in CIA blank control, however it was less in the p-T-shRNA group than that in the CIA blank control. p-T-shRNA, T-bet shRNA recombinant plasmid; pIRES-T-bet, plasmid expressing T-bet; IFN- $\gamma$, interferon- $\gamma$; IL-17, interleukin-17; IL-4, interleukin-4; CIA, collagen-induced arthritis; PMA, phorbol myristate acetate; MACS, magnetic-activated cell sorting; qRT-PCR, quantitative real-time PCR; PLN, peripheral lymph node. 
demonstrate that silencing $T$-bet may have an apparent effect on autoreactive T cells and autoimmunity (21-23).

The RNA interference technique has become a convenient and easy way to knock down target genes (24-26). We used the RNA interference technique to silence the target gene $T$-bet in CIA mice in this experiment. Our results revealed a previously unrecognized role of T-bet in promoting the course of the disease and demonstrated that $T$-bet was a critical factor in CIA development. We constructed the eukaryotic plasmid carrying the $T$-bet gene (pIRES-T-bet) to enhance T-bet expression and the small hairpin RNA (p-T-shRNA) to silence $T$-bet expression in local joint tissue. Through increasing or reducing the expression of T-bet in the right hind paw in the experimental mice, we discovered it was effective in reducing $T$-bet expression and hence ameliorated the inflammatory condition at an early stage. The results also indicated that, in the initial stage of CIA, silencing T-bet was able to inhibit the expression of IFN- $\gamma$ and IL-17. These cytokines secreted from lymphocytes or joints produced a significant effect on local inflammation. IFN- $\gamma$ level was closely associated with $T$-bet expression, while IL-17 was regulated by several transcriptional factors, which may crosstalk with $T$-bet. The effect of $T$-bet on the secretion and function of IL-17 was not clear in the present study, although it may be associated with the time point when pIRES-Tbet or p-T-shRNA was injected. In the initial stage of joint inflammation, silencing $T$-bet may relieve arthritis. Hence, we propose that RNA interference to the T-bet gene may be developed as a potential factor to prevent inflammation at an early stage in RA patients.

\section{Acknowledgements}

This study was supported by the National Natural Science Foundation of China (no. 31270947, no. 81072453, no. 31170849), the Natural Science Foundation of Colleges and Universities in Jiangsu Province and Innovation Fund for candidate of doctor in Jiangsu Province (grant no. 09KJB310001 and CXZZ11_0593, respectively).

\section{References}

1. Rodeghero R, Cao Y, Olalekan SA, Iwakua Y, Glant TT and Finnegan A: Location of $\mathrm{CD}^{+}{ }^{+} \mathrm{T}$ cell priming regulates the differentiation of Th1 and Th17 cells and their contribution to arthritis. J Immunol 190: 5423-5435, 2013.

2. Lin GJ, Huang SH, Chen SJ, Wang CH, Chang DM and Sytwu HK: Modulation by melatonin of the pathogenesis of inflammatory autoimmune diseases. Int J Mol Sci 14: 1174211766, 2013.

3. Su Z, Shotorbani SS, Jiang X, Ma R, Shen H, Kong F and Xu H: A method of experimental rheumatoid arthritis induction using collagen type II isolated from chicken sternal cartilage. Mol Med Rep 8: 113-117, 2013.

4. Fox DA, Gizinski A, Morgan R and Lundy SK: Cell-cell interactions in rheumatoid arthritis synovium. Rheum Dis Clin North Am 36: 311-323, 2010.
5. Chabas D, Baranzini SE, Mitchell D, et al: The influence of the proinflammatory cytokine, osteopontin, on autoimmune demyelinating disease. Science 294: 1731-1735, 2001.

6. Szabo SJ, Kim ST, Costa GL, Zhang X, Fathman CG and Glimcher LH: A novel transcription factor, T-bet, directs Th1 lineage commitment. Cell 100: 655-669, 2000.

7. Shier P, Hofstra CL, Ma XJ, Wu Y, Ngo K and Fung-Leung WP: Tbt-1, a new T-box transcription factor induced in activated Th1 and $\mathrm{CD}^{+} \mathrm{T}$ cells. Immunogenetics 51: 771-778, 2000.

8. Hwang ES: Transcriptional regulation of T helper 17 cell differentiation. Yonsei Med J 51: 484-491, 2010.

9. Tesmer LA, Lundy SK, Sarkar S and Fox DA: Th17 cells in human disease. Immunol Rev 223: 87-113, 2008.

10. Shahrara S, Huang Q, Mandelin AM 2nd and Pope RM: TH-17 cells in rheumatoid arthritis. Arthritis Res Ther 10: R93, 2008.

11. Stamp LK, James MJ and Cleland LG: Interleukin-17: the missing link between T-cell accumulation and effector cell actions in rheumatoid arthritis? Immunol Cell Biol 82: 1-9, 2004.

12. Mathur AN, Chang HC, Zisoulis DG, Kapur R, Belladonna ML, Kansas GS and Kaplan MH: T-bet is a critical determinant in the instability of the IL-17-secreting T-helper phenotype. Blood 108: 1595-1601, 2006

13. Yang Y, Weiner J, Liu Y, Smith AJ, Huss DJ, Winger R, Peng $\mathrm{H}$, Cravens PD, Racke MK and Lovett-Racke AE: T-bet is essential for encephalitogenicity of both Th1 and Th17 cells. J Exp Med 206: 1549-1564, 2009.

14. Gocke AR, Cravens PD, Ben LH, Hussain RZ, Northrop SC, Racke MK and Lovett-Racke AE: T-bet regulates the fate of Th1 and Th17 lymphocytes in autoimmunity. J Immunol 178: 1341$1348,2007$.

15. Ortmann RA and Shevach EM: Susceptibility to collagen-induced arthritis: cytokine-mediated regulation. Clin Immunol 98: 109-118, 2001.

16. Scrivo R, Di Franco M, Spadaro A and Valesini G: The immunology of rheumatoid arthritis. Ann NY Acad Sci 1108: 312-322, 2007.

17. Sarkar S and Fox DA: Dendritic cells in rheumatoid arthritis. Front Biosci 10: 656-665, 2005.

18. Modi S, Soejima M and Levesque MC: The effect of targeted rheumatoid arthritis therapies on anti-citrullinated protein autoantibody levels and B cell responses. Clin Exp Immunol 173: 8-17, 2013.

19. Wang J, Fathman JW, Lugo-Villarino G, Scimone L, von Andrian U, Dorfman DM and Glimcher LH: Transcription factor T-bet regulates inflammatory arthritis through its function in dendritic cells. J Clin Invest 116: 414-421, 2006.

20. Lametschwandtner G, Biedermann T, Schwärzler C, Günther C, Kund J, Fassl S, Hinteregger S, Carballido-Perrig N, Szabo SJ, Glimcher LH and Carballido JM: Sustained T-bet expression confers polarized human Th2 cells with Th1-like cytokine production and migratory capacities. J Allergy Clin Immunol 113: 987-994, 2004

21. Lovett-Racke AE, Rocchini AE, Choy J, Northrop SC, Hussain RZ, Ratts RB, Sikder D and Racke MK: Silencing T-bet defines a critical role in the differentiation of autoreactive $\mathrm{T}$ lymphocytes. Immunity 21: 719-731, 2004.

22. Lai Kwan Lam Q, King Hung Ko O, Zheng BJ and Lu L: Local BAFF gene silencing suppresses Th17-cell generation and ameliorates autoimmune arthritis. Proc Natl Acad Sci USA 105: 14993-14998, 2008

23. Bettelli E, Sullivan B, Szabo SJ, Sobel RA, Glimcher LH and Kuchroo VK: Loss of T-bet, but not STAT1, prevents the development of experimental autoimmune encephalomyelitis. J Exp Med 200: 79-87, 2004.

24. Hannon GJ: RNA interference. Nature 418: 244-251, 2002.

25. Denli AM and Hannon GJ: RNAi: an ever-growing puzzle. Trends Biochem Sci 28: 196-201, 2003.

26. Escobar T, Yu CR, Muljo SA and Egwuagu CE: STAT3 activates miR-155 in Th17 cells and acts in concert to promote experimental autoimmune uveitis. Invest Ophthalmol Vis Sci 54: 4017-4025, 2013. 